\title{
MORSE THEORY FOR FIXED POINTS OF SYMPLECTIC DIFFEOMORPHISMS
}

\author{
ANDREAS FLOER
}

\begin{abstract}
We prove the following special case of the Arnold conjecture on the fixed points of an exact deformation $\varphi$ of a compact closed symplectic manifold $P$ : If $\pi_{2}(P)=0$ and all fixed points of $\varphi$ are nondegenerate, then their number is greater than or equal to the sum of the Betti numbers of $P$ with respect to $Z_{2}$ coefficients.
\end{abstract}

Let $P$ be a symplectic manifold, i.e. $P$ is a smooth manifold equipped with a closed and nondegenerate 2 -form $\omega$. Then we can assign to each smooth function

$$
H: P \times \mathbf{R} \rightarrow \mathbf{R} ; \quad H(x, t)=H_{t}(x)
$$

a family $X_{t}$ of vector fields on $P$ defined by $\omega\left(\cdot, X_{t}\right)=d H_{t}$. This vector field is called the (exact) Hamiltonian vector field associated with the (timedependent) Hamiltonian $H$. If $P$ is compact, then the differential equation

$$
\frac{d}{d t} \varphi_{H, t}(x)=X_{t}\left(\varphi_{H, t}(x)\right)
$$

with initial condition $\varphi_{H, 0}(x)=x$ defines a family of smooth diffeomorphisms of $P$, which also preserve the symplectic structure, i.e. for each $t \in \mathbf{R}$ we have $\varphi_{t}^{*} \omega=\omega$. In fact, the set

$$
D=\left\{\varphi_{H, t} \mid t \in \mathbf{R} \text { and } H \in C^{\infty}(P \times \mathbf{R})\right\}
$$

of exact diffeomorphisms turns out to be a subgroup of the group of symplectic diffeomorphisms on $P$.

Since each $\varphi \in D$ is homotopic to the identity, the Lefschetz fixed point theorem implies that if all fixed points $x$ of $\varphi$ are nondegenerate in the sense that

$$
\operatorname{det}(D \varphi(x)-\mathrm{id}) \neq 0,
$$

then the sum of the signs of (4) over all fixed points of $\varphi$ is equal to the Euler characteristic $\chi(P)$. In particular, if all fixed points are nondegenerate, their number must be equal to or greater than the absolute value of $\chi(P)$. It has been conjectured by $V$. Arnold that a stronger result holds for exact diffeomorphisms: the number of fixed points of each $\varphi \in D$ should satisfy estimates similar to those obtained by Morse theory for the number of critical points of a smooth function on $P$.

Received by the editors August 1, 1986 and, in revised form, November 5, 1986.

1980 Mathematics Subject Classification (1985 Revision). Primary 53C15; Secondary $58 \mathrm{~F} 05$.

(C)1987 American Mathematical Society $0273-0979 / 87 \$ 1.00+\$ .25$ per page 
Results in this direction have been proved in [2] for the standard symplectic structure on the even-dimensional torus, in [3 and 7] for surfaces and other hyperbolic manifolds and in [4] for the complex projective space. Moreover, there has been a perturbation result for general symplectic manifolds, see [8]. Recently, Gromov [5] proved the existence of at least one fixed point for any exact deformation on $P$ provided that $\pi_{2}(P)=0$. In this note, we announce the extension of this existence result to a Morse theory of nondegenerate fixed points.

THEOREM 1. Let $P$ be a compact closed symplectic manifold with $\pi_{2}(P)=$ 0 . Let $\varphi: P \rightarrow P$ be an exact diffeomorphism all of whose fixed points are nondegenerate. Then the number of fixed points is greater than or equal to the sum of the Betti numbers of $P$ with respect to $Z_{2}$-coefficients.

It is conceivable that the ideas underlying the proof of Theorem 1 also work in the case of a general symplectic manifold. The estimate is a consequence of the following more precise relation between the fixed point set and the cohomology of $P$.

THEOREM 2. With $P$ and $\varphi$ as in Theorem 1 , let $C^{*}$ denote the $Z_{2}$-vector space over the set of fixed points of $\varphi$. Then there exists a homomorphism

$$
\delta: C^{*} \rightarrow C^{*}
$$

of $Z_{2}$-vector spaces so that $\delta \delta=0$ and so that

$$
\operatorname{ker} \delta / \operatorname{im} \delta=H^{*}\left(P, Z_{2}\right) \text {. }
$$

The coboundary operator $\delta$ is constructed as follows. For $\varphi \in D$, define

$$
\Omega(\varphi)=\left\{z \in C^{\infty}([0,1], P) \mid z(1)=\varphi(z(0))\right\} .
$$

Moreover, choose an almost complex structure $J$ so that the bilinear form $g=\omega(J \cdot, \cdot)$ is a metric, i.e. is symmetric and positive. Then we consider formally the flow generated by the "vector field" $V(z)=J \dot{z}$ on $\Omega(\varphi)$. To be more precise, we consider 1-parameter families $u: \mathbf{R} \times[0,1] \rightarrow P$ in $\Omega$ satisfying

$$
\frac{\partial u(\tau, t)}{\partial \tau}+J(u(\tau, t)) \frac{\partial u(\tau, t)}{\partial t}=0 .
$$

Clearly, the fixed points of this "flow" are in 1-1 correspondence with fixed points of $\varphi$. We are particularly interested in the sets $\mathcal{M}(x, y)$ of solutions of (8) converging to fixed points $x$ and $y$ for $\tau \rightarrow \pm \infty$. Applying a small perturbation to the almost complex structure $J$ if necessary, we find that these sets are smooth finite-dimensional manifolds. Moreover, the group $\mathbf{R}$ acts freely on $\mathcal{M}(x, y)$ by translation in the first variable. We now define

$$
\langle x, \delta y\rangle= \begin{cases}\#(\mathcal{M}(x, y) / \mathbf{R} \bmod 2 & \text { if } \operatorname{dim} \mathcal{M}(x, y)=1, \\ 0 & \text { otherwise }\end{cases}
$$

For an analogous construction in finite-dimensional Morse theory, see Milnor [6]. In order for (9) to be well defined, we need a compactness property of holomorphic curves, see also [5]. 
It turns out that (9) defines the matrix elements of an operator $\delta$ satisfying $\delta \delta=0$. In order to show that it also satisfies (6), we show that the quotient $\operatorname{ker} \delta / \operatorname{im} \delta$ is invariant under deformation of $\varphi$ within $D$. By the definition of $D$, we can now deform $\varphi$ into the identity. The relation (6) is then proved by a perturbation argument.

The author wishes to thank M. Gromov, A. Weinstein, and E. Zehnder for valuable discussions.

\section{REFERENCES}

1. V. I. Arnold, Mathematical methods of classical mechanics, (Appendix 9), SpringerVerlag, Berlin and New York, 1978.

2. C. C. Conley and E. Zehnder, The Birkhoff-Lewis fixed point theorem and a conjecture by V. I. Arnold, Invent. Math. 73 (1982), 33-49.

3. A. Floer, Proof of the Arnold conjecture for surfaces and generalizations for certain Kähler manifolds, Duke Math. J. 53 (1986), 1-32.

4. B. Fortune, A symplectic fixed point theorem for $\mathbf{C} P^{n}$, Invent. Math. 81 (1985), 29-46.

5. M. Gromov, Pseudoholomorphic curves in symplectic manifolds, Invent. Math. 82 (1985), 307-347.

6. J. Milnor, Lectures on the $H$-cobordism theorem, Mathematical Notes, Princeton Univ. Press, 1965.

7. J. C. Sikorav, Points fixes d'un symplectomorphisme homologue de l'identité, J. Differential Geom. 22 (1985), 49-79.

8. A. Weinstein, $C^{0}$-perturbation theorems for symplectic fixed points and Lagrangian intersections, Lecture Notes, Amer. Math. Soc. Summer Institute on nonlinear functional analysis and applications, Berkeley, 1983.

Department of Mathematics, State University of NeW York at Stony BROOK, STONY BROOK, NEW YORK 11794

Current address: Courant Institute, New York University, 251 Mercer Street, New York, New York 10012 